\title{
Revealing the Influence Mechanism of Urban Built Environment on Online Car-Hailing Travel considering Orientation Entropy of Street Network
}

\author{
Sai Wang $\mathbb{D}^{1},{ }^{1}$ Jianjun Wang $\left(\mathbb{D},{ }^{1}\right.$ Weijia $\mathrm{Li}^{2}{ }^{2}$ Jialin Fan, ${ }^{1}$ and Mingyu Liu ${ }^{1}$ \\ ${ }^{1}$ College of Transportation Engineering, Chang'an University, Xi'an 710064, China \\ ${ }^{2}$ School of Architecture, Chang'an University, Xi'an 710064, China \\ Correspondence should be addressed to Jianjun Wang; 2019021059@chd.edu.cn
}

Received 26 August 2021; Revised 23 November 2021; Accepted 12 January 2022; Published 18 February 2022

Academic Editor: Giulio E. Cantarella

Copyright (c) 2022 Sai Wang et al. This is an open access article distributed under the Creative Commons Attribution License, which permits unrestricted use, distribution, and reproduction in any medium, provided the original work is properly cited.

Exploring the impact of the urban built environment on online car-hailing, an increasingly popular mode of urban transportation, is crucial for developing corresponding transportation strategies and addressing sustainable urban planning. This paper captures that the form and orientation of the neighborhood network have a certain degree of influence on travel behavior. Therefore, the road network orientation entropy indicator is introduced based on the originally built environment elements, and the orientationorder $(\mathrm{OO})$ indicator calculation model is constructed to normalize it. This study further tests and verifies its impact on the travel behavior of online car-hailing, thereby improving the factor characterization of neighborhood design latitude in the urban built environment. From the perspective of spatial correlation and spatial heterogeneity, the optimal models are selected by model comparative analysis, namely, the spatial Durbin model (SDM) and the mixed geographically weighted regression model (MGWR). Based on the optimal model estimation results, the influence mechanism of the urban built environment on the spatialtemporal distribution of online car-hailing travel in three cases (considering road network density (RD), considering RD and OO, considering $\mathrm{OO}$ ) is compared and analyzed. The results show that the model fitting considering the OO is the best in the above three cases, and the OO has a significant impact on DiDi travel compared with the RD in this study, which verifies the rationality and necessity of selecting the $\mathrm{OO}$ in this study. In addition, catering service, corporate business, and OO have significant positive spillover effects, while the spillover effects of sports and leisure service and land-use mix are negative. The indicators of the bus station, residential district, catering service, shopping service, corporate business, land-use mix, life service, and OO have significant spatial heterogeneity.

\section{Introduction}

In recent years, with the continuous expansion of the urban scale, it is vital to excavate the influence of the urban built environment on traffic travel. Built environment refers to the human environment constructed and configured to provide the needs of human activities, including the spatial environment formed by the interaction of multiple factors such as land-use form, transportation infrastructure, and urban design [1]. At present, "five Ds" indicators are mostly used to characterize the built environment, including density, diversity, design, destination accessibility, and distance to transit [2]. Based on the development background of big data technology, online car-hailing emerges as a new business model with its convenient and flexible booking service. The development of online car-hailing can integrate idle vehicle resources and improve the utilization rate of vehicles, which eases the difficulty of people taking taxis to a certain extent and conforms to the development concept of sharing economy. However, the rapid development of the emerging transportation mode will also bring corresponding difficult challenges to urban traffic planning and management. Such challenges mainly include how to guide and manage the development of DiDi travel, how to integrate it into a variety of transportation systems (e.g., cars, buses, taxis, subways, and nonmotorized traffic), and how to 
reasonably integrate the built environment policies (e.g., regional development planning, comprehensive land development, and street network construction) with transportation policies (e.g., relevant policies such as bus, taxi, and online car-hailing operation management) [3], which are also issues that researchers need to focus on at present.

Related studies have shown that the built environment is significantly correlated with the travel behavior of online car-hailing [4-9]. However, different researchers often select different indicators to measure the built environment. For example, in previous studies, the neighborhood design dimension in "five Ds" is often characterized by factors such as road network density or intersection density. When the above density is small and the impact on travel behavior is not significant, researchers believe that the impact of neighborhood design on travel behavior is not significant [3]. However, the form and orientation of the neighborhood network are crucial to defining the spatial logic and order of the city, and this feature of neighborhood design is not taken into account in the above analysis. Therefore, it is necessary to find another indicator to reflect the orientation and order of the neighborhood to further improve the representation of the dimension of the neighborhood design. In addition, the influence of environmental variables may vary with the urban spatial forms, and neglecting the spatial influence may lead to inaccurate results. Thus, when modeling and analyzing the impact of the built environment on travel behavior, it is necessary to further consider the influence of spatial dependence (spatial autocorrelation) and spatial structure inhomogeneity (spatial heterogeneity) so as to more accurately grasp the impact mechanism of urban form factors on online car-hailing travel [10-12].

Therefore, this study focuses on the selection of urban built environment measurement indicators and the selection and analysis of modeling methods, specifically including three main tasks. Firstly, based on the principle of the street network model and the quantification and visualization of street network orientation entropy by Mohajeri and Gudmundsson [13], we use OpenStreetMap software to construct the calculation model of orientation-order indicator to improve the selection of independent variables of the urban built environment, that is, taking orientation entropy as one of the characterization factors of independent variables of the built environment. Secondly, this study constructs models from two different perspectives of spatial correlation and spatial heterogeneity using the DiDi travel data of Chengdu released by DiDi and POI data collected by Gaode Map, selects the models with a higher fitting degree, and then determines the factors that can better reflect the characteristics of neighborhood design. Finally, the modeling results are analyzed to explore the spatial and temporal distribution of DiDi travel, which provides a theoretical basis for reducing the empty driving rate of DiDi trips, optimizing the operation of the urban road network, and forecasting the spatial demand of DiDi travel.

The remainder of this paper is organized as follows. Section 2 reviews relevant literature studies. Section 3 describes the data and variables used in this study. The construction of spatial correlation and heterogeneity models is described in detail in Section 4. In Section 5, the optimal models of spatial correlation and spatial heterogeneity are selected, the estimation results of the optimal models are presented in detail, and the influences of built environment indicators (especially the orientation order) on DiDi travel are discussed. The final section summarizes the main findings and proposes future research directions.

\section{Literature Review}

2.1. Studies on the Relationship between the Built Environment and Travel Behavior of Online Car-Hailing. For online carhailing, previous studies have focused on the characteristics and preferences of passengers $[14,15]$, the matching degree of online car-hailing services with passengers [16], scheduling strategies [17], and so on. In recent years, scholars have begun to follow the influence of the built environment on the travel behavior of online car-hailing, which is mainly reflected in two study areas. Most of the studies were based on the data of online car-hailing in the United States. For example, Alemi et al. [4] used the California Millennials Dataset to prove that land-use diversity and destination accessibility have significant impacts on the demand for Uber. Yu and Peng $[5,6]$ divided RideAustin data into two types: weekday travel data and weekend travel data. It is found that population density, sidewalk density, land-use mix, and traffic accessibility are positively correlated with ride demand. Sabouri et al. [7] illustrated that the demand for Uber in New York was positively correlated with the land-use mix, population density, and transit travel density. Another few studies were based on the data of DiDi travel in the local area of the Second Ring Road of Chengdu, China. For instance, Li et al. [3] demonstrated that the distance to CBD and the mixing degree of land use were positively correlated with $\mathrm{DiDi}$ ride-hailing and argued that recreation and entertainment POI and residential district POI were the main influencing factors for nighttime ride-hailing. With the promotion of sharing travel, the carpooling services approach to traffic has become popular, and ridesplitting orders can be further extracted from DiDi orders. Related studies have confirmed that the distance to the CBD and the land-use diversity affect the ridesplitting rate $[8,9]$.

Given the studies on the impact of the built environment on online car-hailing travel behavior, we find that, in previous studies, the measurement factors (e.g., road network density or intersection density) of neighborhood design dimension have three types of effects on online car-hailing travel. First, road network density or intersection density has a significant positive impact on travel behavior. For example, $\mathrm{Yu}$ and Peng $[5,6]$ found that higher road density could attract more online car-hailing travel, mainly "because of higher exposure to available ridesourcing vehicles on the road and less response." Second, contrary to their findings, Sabouri et al. [7] indicated that the intersection density was negatively correlated with Uber travel demand, which might be attributed to the relative advantages of other transportation modes, such as private cars, buses, pedestrians, or bicycles in areas with denser street networks. Third, Li et al. [3] found that the road network density had no appreciable impact on the boarding ridership of online car-hailing. The 
possible reason is that the selection of appropriate variables should be based on local conditions rather than simply transplanting those.

In summary, there are two main problems in current studies on online car-hailing. First of all, the influence of all dimensions (namely, "five Ds") of the built environment is not fully controlled when selecting variables in the above study, which may lead to deviation in the degree of influence of the built environment on online car-hailing travel. Secondly, when the influence of road network density on online car-hailing travel is not obvious, it is necessary to further find and verify new indicators to characterize neighborhood design so as to more accurately determine whether it has an impact on travel behavior. Therefore, to accurately measure the impact of the built environment on online car-hailing travel behavior, it is necessary to further improve the selection of built environment measurement factors.

\subsection{Studies on Orientation Entropy of Street Network.} Spatial networks, such as streets, paths, and traffic routes, organize human travel in complex urban systems, which shape travel behavior, location decisions, and urban fabric. In particular, the form and orientation of street networks help to define the spatial logic and order of the city $[18,19]$. Previous studies usually used geographic information systems (GIS) to directly measure geometric grid, neighborhood, and street attributes, such as street connectivity, density, and intersection types [20]. Accordingly, researchers have recently focused more on the patterns, performance, complexity, and configuration of street networks. A stream of research has explored the nature of entropy and order in urban street networks, attempting to quantify the spatial order and disorder patterns of the urban circulatory system. It showed that entropy measures can well reveal the order and disorder of urban streets, in which the order refers to a higher degree of the grid and has better connectivity, while the disorder is vice versa $[13,21]$. Furthermore, scholars often use entropy to study the order of street networks, quantify street orientation, and evaluate the degree of order by visualizing how the street network follows the geometric logic of a single grid. Barthelemy et al. [22] revealed that entropy has a profound theoretical connection with the complexity of urban blocks in the urban blocks spatial network, which will have a certain impact on the travel behavior of urban traffic. Further, the spatial order of the urban order system can be quantified by exploring the essence of urban street network entropy [23]. Based on the study of circuity, order, and entropy, Boeing [24] investigated cities around the world using OpenStreetMap data, explored the patterns and relationships of the street networks, and obtained the network structure of different cities along the process of human interaction and transportation. In addition, it is suggested that future work can consider the importance of taking multiple indicators in concert to study their impact on travel behavior.

Through the above studies, it can be seen that street network orientation entropy has an impact on travel behavior. Therefore, the spatial logic and geometric order generated by the orientation of the street network should be considered when analyzing the impact of the built environment on online car-hailing behavior; that is, the orientation entropy is taken as one of the factors to measure the neighborhood design in "five Ds," and then the degree of its influence on travel behavior is measured.

2.3. Studies on Spatial Correlation and Heterogeneity. To explore the relationship between the built environment and travel behavior, the traditional methods are mainly ordinary least squares (OLS) [25] and the discrete choice model in the four-stage method (e.g., logit models [4] and probit models [26]), but the above models can only be used for global estimation without considering the spatial properties [27]. However, travel behavior is highly sensitive to the urban built environment and will change with spatial change. Ignoring spatial instability may lead to inconsistent parameters or inaccurate test results. To further study the spatial correlation and spatial heterogeneity of the built environment on travel behavior, scholars have used the spatial econometric model to explore the following. On the one hand, considering the spatial dependence of travel demand, scholars have used the spatial correlation model $[28,29]$ (e.g., spatial autoregressive (SAR) model; spatial Durbin model (SDM); spatial error model (SEM)) to capture the direct and spatial spillover effects of the built environment on travel demand. Chen et al. compared and analyzed the impact of the urban built environment on taxi travel demand based on OLS, SAR, SDM, and SEM models and obtained the optimal fitting result of the SDM model; that is, this method can not only capture the spatial interaction between dependent variables and independent variables but also consider the spatial correlation of explanatory variables, namely, spatial spillover effect [29]. On the other hand, considering the impact of spatial heterogeneity on travel, relevant researchers have adopted the spatial heterogeneity correlation models (e.g., geographically weighted regression (GWR); multiscale geographically weighted regression (MGWR)), which effectively revealed the spatial variation of the influencing coefficients of various influencing factors in the study region [30, 31]. For instance, Li et al. [3] used the GWR model to explore different impacts on spatial dimensions, and $\mathrm{Yu}$ and Peng [5] further constructed a geographically weighted regression Poisson distribution model (GWPR) to analyze the spatial heterogeneity in different periods (morning peak, subpeak, and evening peak).

Through the above summaries, it can be seen that current studies only consider one of the above two aspects, while this study uses the same data to contain both the spatial dependence and spatial heterogeneity of travel behavior and more accurately determine the impact degree of the built environment on online car-hailing travel.

\section{Data Preparation}

3.1. Study Area and Data Source. This study selected the DiDi trajectory data and order data of the local area of the Second Ring Road in Chengdu, China (longitude: 30.65-30.72; 
latitude: 104.04-104.12), from November 1 to November 30, 2016. The trajectory dataset comes from the DiDi Gaia Data Opening Plan, including driver ID, order ID, timestamps, longitude, and latitude; the order data contains the order ID, start and end timestamps, and longitude and latitude of pick-up and drop-off locations. The study area of this paper is consistent with the data coverage. To study the impact of the built environment on online car-hailing travel on a fine scale, the study area was categorized into 289 grids with a $0.5 \mathrm{~km} * 0.5 \mathrm{~km}$ grid as the analysis unit, and then the GPS data of pick-up points were subject to coordinate transformation and map matching. The grid division of the study area is shown in Figure 1(a).

The geographical location information of the study area is as follows. The study area mainly includes Jinniu District, Qingyang District, Jinjiang District, and Chenghua District, as indicated in Figure 1(b). Jinniu District, located in the northwest of Chengdu, is the central district of Chengdu and has the north railway station of Chengdu, which is the largest comprehensive transportation hub in the southwest of China. Qingyang District is located in the west of Chengdu, with famous scenic spots such as Qingyang Palace, Kuanzhai Alleys, and business district. The urban land is mainly used for commercial and residential purposes, with a relatively dense road network and developed public transportation facilities and the Chengdu west railway station located in this area. Jinjiang District, located in the southwest of Chengdu, is a historic and prosperous business district with a high degree of land-use mix because it has the East Street known as "the first street of Shu" and the Chunxi Road known as "the Centennial Golden Street." Chenghua district is located in the eastern part of Chengdu's central city, most of which are outside the Second Ring Road. Compared with the other three districts, this district has lower road network density and less number of public transport facilities.

3.2. Dependent Variable Analysis. To explore the built environment factors affecting online car-hailing travel, this study selected the traffic volume of each grid as the dependent variable. Firstly, the travel volume of DiDi orders in one month was statistically analyzed, which showed a changing trend with a cycle of seven days, as shown in Figure 2(a). Further, the travel orders of one month were analyzed in different periods, and the average travel volume of each period in a week was calculated, as shown in Figure 2(b). It can be seen that the distribution of hourly travel volume on weekdays (Monday to Friday) is relatively close, including four peak travel periods, namely, 8:00 to 9: $00,13: 00$ to $14: 00,16: 00$ to $17: 00$, and $20: 00$ to $21: 00$. The travel volume from 0:00 to 5:00 on rest days (Saturday and Sunday) was more than that on weekdays, and the travel peak was delayed until 13:00, which may be due to more free time on weekends and people's travel time being relatively free.

To dissect the travel behavior of DiDi from the finegrained level, this paper mainly dissects the travel behavior of DiDi on weekdays, so the average daily travel volume data on weekdays were selected to analyze the temporal and spatial distribution of passenger flow, as illustrated in Figures 2(c) and 2(d). The hot spots of passenger flow travel were mainly concentrated in the vicinity of Chunxi Road and Wanda Plaza in Jinjiang District and Qingyang District within the study area, and the passenger flow was small in the evening, increased sharply in the morning, and then reached the peak at noon. It could be inferred that the impact of the urban built environment on online car-hailing travel may change with time and space. A large travel volume during the peak period has the greatest impact on the overall travel of the road network compared to the off-peak period, and the current policy measures are mostly carried out based on the research results during the peak period. Therefore, to further study the above space-time evolution rules, the monthly average DiDi travel volume during the peak period (13:00 to $14: 00)$ was selected in this paper.

3.3. Independent Variable Analysis. Based on the analysis of travel characteristics of dependent variables, this study found that the peak time of DiDi's travel volume is different from that of other travel modes (e.g., cars and taxis) from 13: 00 to $14: 00$. At this time, the overall travel condition of road traffic was relatively smooth compared with the peak travel in the morning and evening of the city, which may lead to the phenomenon that road network density or intersection density has no significant impact on online car-hailing travel. At this time, it is necessary to improve the attention of neighborhood orientation and order and test its impact on the travel behavior of online car-hailing. Therefore, based on only selecting road network density or intersection density to characterize neighborhood design, this study drew on the principle of orientation entropy, normalized it to obtain orientation-order indicator, and tested and verified its influence on travel behavior so as to measure the characterization factors of neighborhood design more accurately. In this study, nineteen elements were selected to represent the "five Ds" indicators of the built environment and were used as independent variables in subsequent modeling research, as detailed in Table 1.

\subsubsection{Density and Destination Accessibility Indicator} Selection. Density mainly includes population density and thirteen POIs data. POIs data incorporate residential district, catering service, shopping service corporate business, sports and leisure service, science zone, public facility, finance and insurance services, education and culture, life service, medical and health, government and administration, and accommodation service. Bus station POI and distance to $\mathrm{CBD}$ were used to represent the distance to transit and the destination accessibility, respectively.

3.3.2. Diversity Indicator Selection. The land-use mix was taken as the measurement of land-use diversity, and the calculation formula was obtained by applying the principle of the Herfindahl Hirschman Index, as shown in formula (1). 


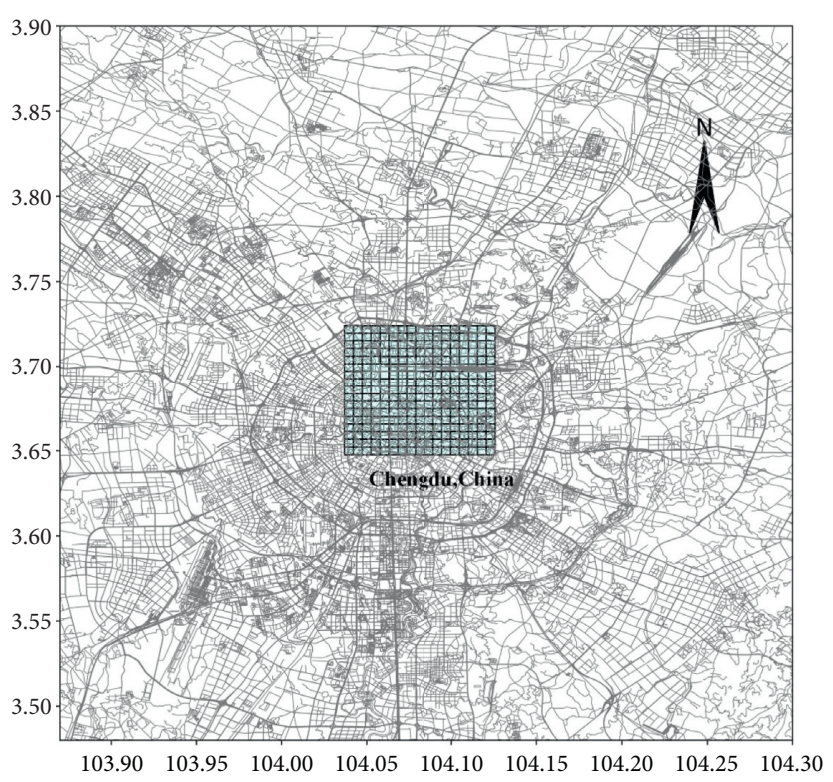

(a)

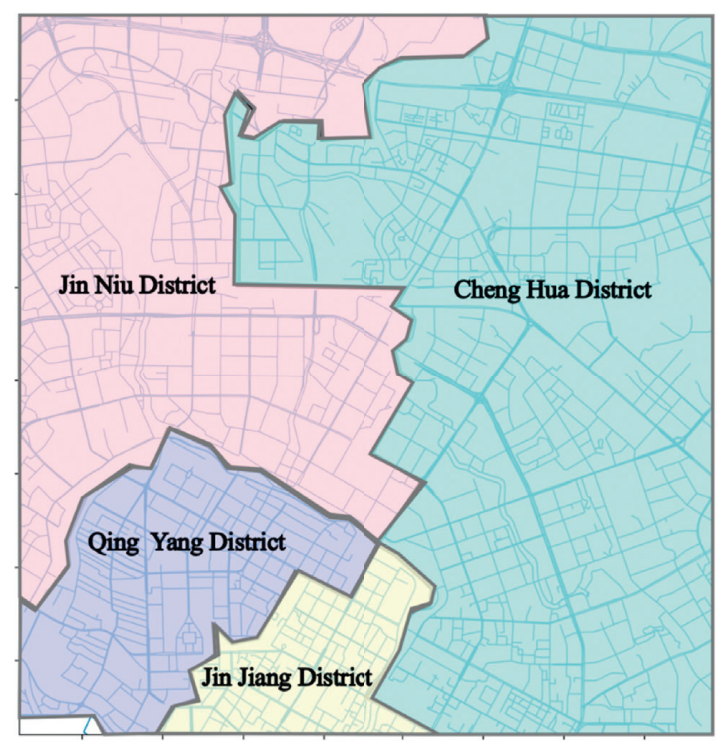

(b)

FIGURE 1: Study area. (a) The geographic location and spatial grids division of the study area. (b) The administrative divisions of the study area.

$$
L M_{i}=\sum_{j=1}^{m}\left(N_{i j} / N_{i}\right)^{2},
$$

where $N_{i}$ is the total POIs numbers in grid $i, N_{i j}$ is the total POIs numbers of class $j$ in grid $i$, and $m$ is the category of POIs.

3.3.3. Neighborhood Design Indicator Selection. The selection of block design indicators in this section mainly includes the following two parts:

(1) Orientation entropy calculation: this study relied on Shannon's entropy principle [24], the street orientation was divided into 36 categories (boxes), the true north was set to $0^{\circ}$, and 36 intervals were created at an interval of $10^{\circ}$. If the street orientation falls in the interval, the orientation is adjudged to be in that interval, which could more intuitively determine the degree of blocks disordered; that is, the smaller the orientation entropy, the higher the degree of gridding and the better the connectivity and street orientation order, and vice versa. The calculation of orientation entropy was based on python's OSMnx toolkit, from which the spatial data, including city boundaries and streets, can be downloaded from OSM and normalized into the dimensionless natural unit. The calculation formula of orientation entropy is shown in formula (2), and its schematic diagram is displayed in Figure 3.

$$
H_{o}=-\sum_{k=1}^{n} P\left(o_{k}\right) \ln P\left(o_{k}\right) \text {, }
$$

where $H_{0}$ represents the street orientation entropy, $n$ is the total number of boxes, $k$ refers to boxes $k$, and $P\left(o_{k}\right)$ refers to the proportion of streets with orientation $k$.

(2) Orientation-order indicator calculation: the orientation-order indicator was obtained by normalizing the orientation entropy, which means linearizing the location of the city from complete disorder (uniform distribution) to perfect order (grid distribution), making its value between 0 and 1 to achieve the effect of normalization. The calculation formula is as follows:

$$
\varphi=1-\left(\frac{H_{0}-H_{g}}{H_{\text {max }}-H_{g}}\right)^{2},
$$

where $H_{\max }$ is the maximum entropy; that is, the street bearing is perfectly and uniformly distributed in 36 intervals of the street, and its value is $3.584 ; \mathrm{H}_{g}$ represents the ideal grid entropy, and the blocks are arranged in four equal proportions (e.g., east-southwest-north), with a value of 1.386 .

If the orientation order $\varphi=0$, it indicates the uniform distribution of streets in all orientations; if $\varphi=1$, it means that the streets appear as a perfectly ordered grid. That is, the larger the orientation-order indicator, the higher the degree of grid and connectivity of the block and the better the direction order of the street, and vice versa. Relevant studies have shown that considering the four indicators, namely, orientation order, average circuity, average node degree, and median street segment length, can present the characteristics of the street network. In addition, the orientation-order 


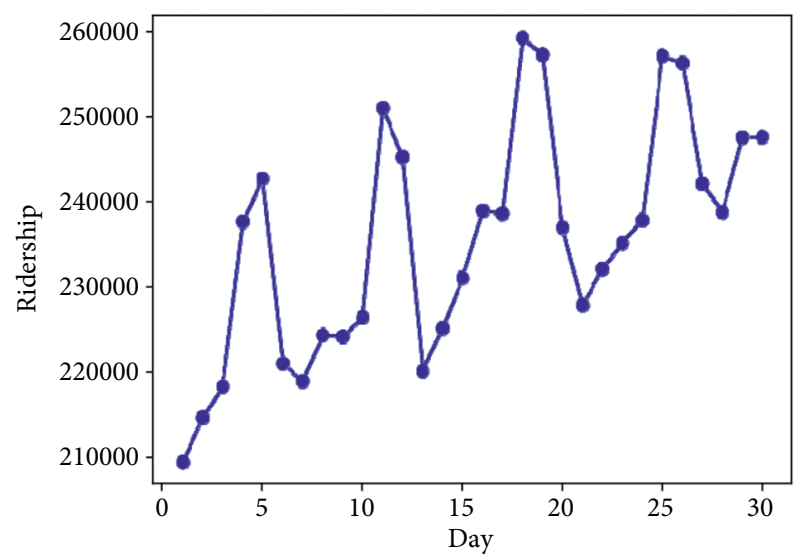

(a)

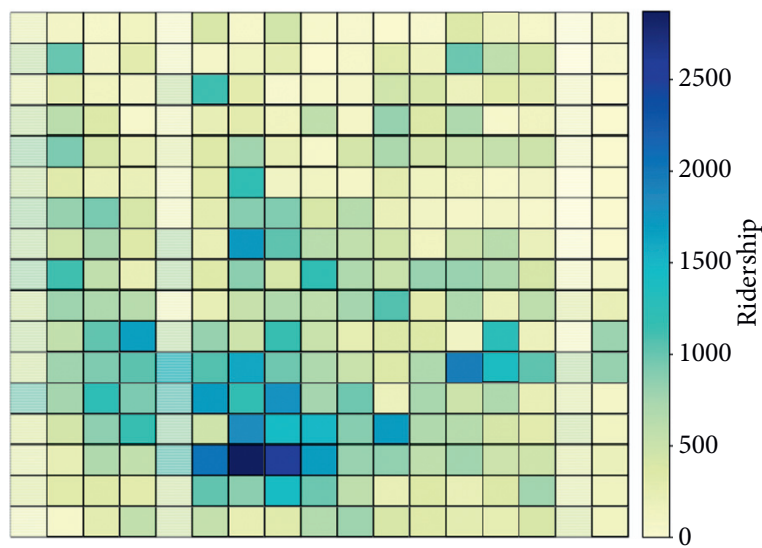

(c)

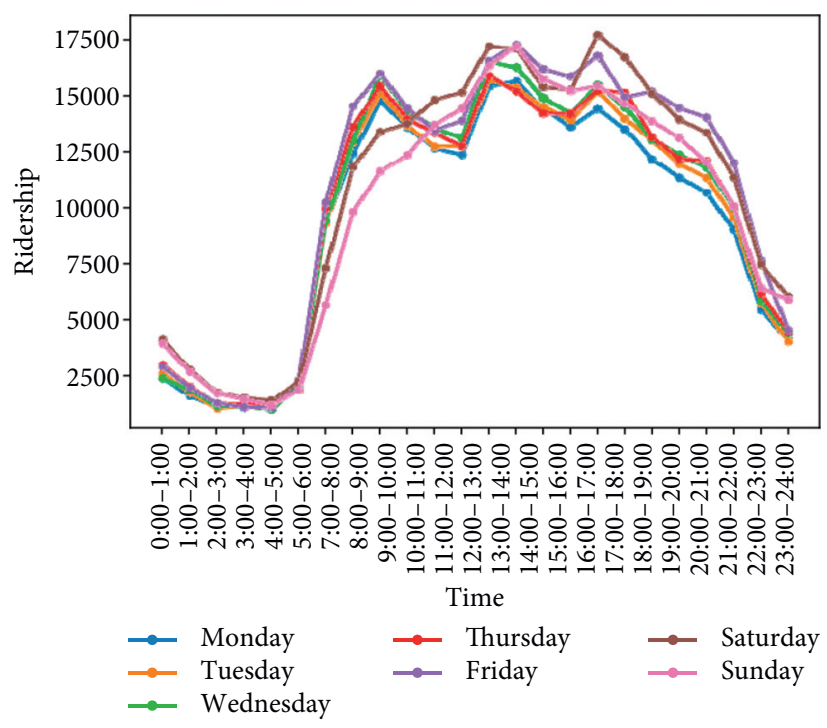

(b)

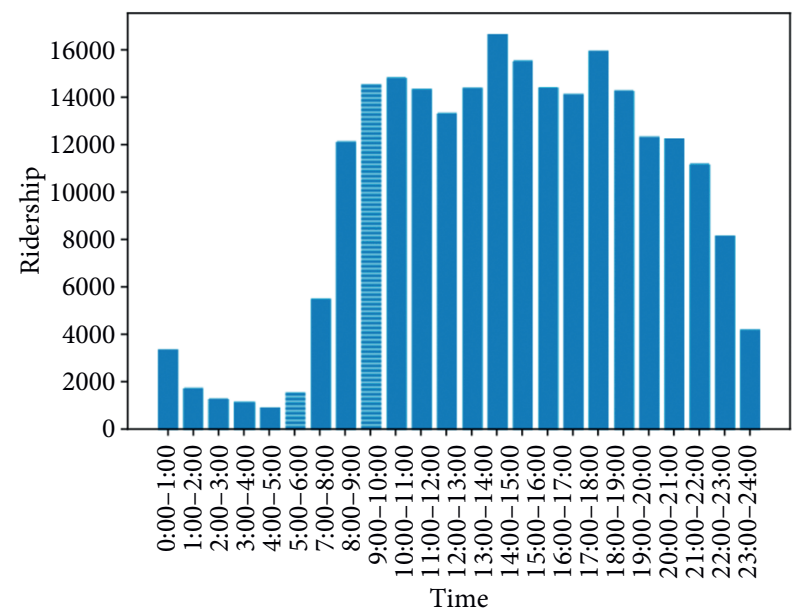

(d)

Figure 2: Travel distribution map of traffic volume. (a) Distribution of monthly travel volume. (b) Distribution of weekly travel volume. (c) Spatial distribution of average daily travel. (d) Spatial distribution of average daily travel.

TABLE 1: Independent variable selection of "five Ds" indicators of the built environment.

\begin{tabular}{|c|c|c|c|}
\hline "Five Ds" indicators & $\begin{array}{l}\text { Main factors selected of } \\
\text { previous studies }\end{array}$ & Factors selected of this study & Data source \\
\hline \multirow{2}{*}{ Density } & \multicolumn{2}{|c|}{ People density (PD) } & $\begin{array}{c}\text { The sixth nationwide population } \\
\text { census }\end{array}$ \\
\hline & \multicolumn{2}{|c|}{ Thirteen POIs (RD, CS, SS, CB, SL, SZ, PF, FI, EC, LS, MH, GA, and AS) } & Gaode Map \\
\hline $\begin{array}{l}\text { Destination } \\
\text { accessibility }\end{array}$ & \multicolumn{2}{|c|}{ Distance to CBD (DC) } & Gaode Map \\
\hline Diversity & \multicolumn{2}{|c|}{ Land-use mix (LM) } & Calculation of POIs data \\
\hline Distance to transit & \multirow{2}{*}{$\begin{array}{l}\text { Distance to the bus station } \\
\text { Road network density or } \\
\text { intersection density }\end{array}$} & \multirow{2}{*}{$\begin{array}{c}\text { Bus station POI (BS) } \\
\text { Orientation order (OO) and road network } \\
\text { density (RD) }\end{array}$} & Gaode Map \\
\hline Design & & & OpenStreetMap (OSM) \\
\hline
\end{tabular}

indicator is significantly correlated with the other three spatial order indicators of the above four indicators (the orientation-order is moderately negatively connected with the average circuity and median street length and moderately positively correspond with the average node degree) $[10,23,24]$. Therefore, this study selected the orientation-order indicator as one of the characterization factors of neighborhood design. 


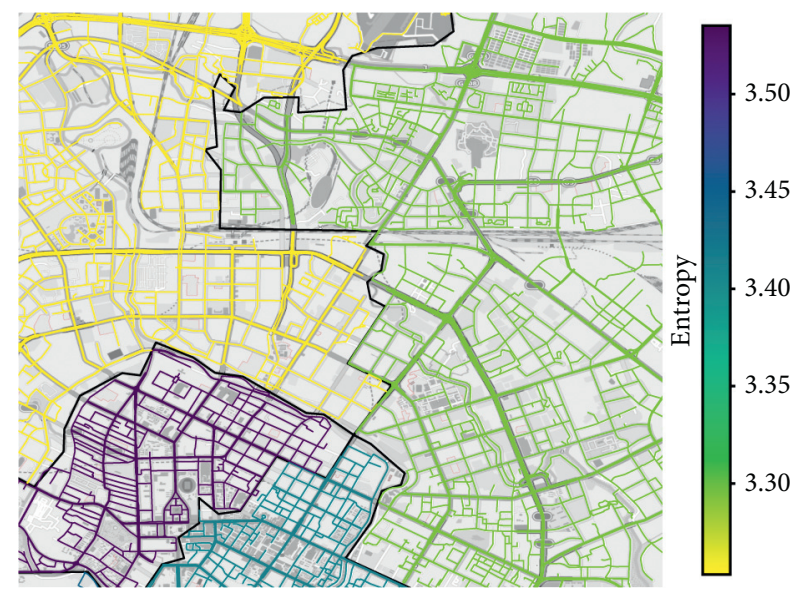

Figure 3: Diagram of orientation entropy of road network.

Through the above analysis, the heatmaps of the respective variables selected in this paper are shown in Figure 4.

\section{Methodology}

4.1. Spatial Correlation Model Construction. To explore the influence of spatial correlation, the spatial nonfixed effects caused by the changes of independent variables due to the different study regions were considered, and the spatial correlation models were established to clarify the spatial direct effect and spillover effect of the built environment on demand for DiDi travel.

4.1.1. Model Construction. This part aims to explore the impact of the urban built environment on the spatial correlation of online car-hailing. The general form of the spatial econometric model adopted is as follows $[29,32]$ :

$$
\left\{\begin{array}{l}
Y_{i}=\lambda \sum_{j=1}^{n} W_{i j} Y_{i}+\theta \sum_{j=1}^{n} W_{i j} X_{i}+\beta X_{i}+\xi, \\
\xi=\gamma \sum_{j=1}^{n} W_{i j} \xi+\varepsilon, \varepsilon \sim N\left(0, \sigma^{2} I_{n}\right),
\end{array}\right.
$$

where $Y_{i}$ represents the $\mathrm{DiDi}$ travel volume within traffic zone $i ; X_{i}$ is the built environment variable in traffic zone $i$; $W_{i j}$ is the spatial weight matrix; $\sum_{j=1}^{n} W_{i j} Y_{i}, \sum_{j=1}^{n} W_{i j} X_{i}$, and $\sum_{j=1}^{n} W_{i j} \xi$ are the spatial lag term of the explained variable, the explanatory variable, and the error property, respectively; $n$ is the number of traffic zones; $\lambda$ and $\theta$ are the spatial correlation coefficients of explained variables and explanatory variables, respectively; $\beta$ is the independent variable regression coefficient; $\gamma$ is the autocorrelation coefficient of spatial error; $\xi$ is a random error term; $\varepsilon$ is a random perturbation term.

Based on the general form of the spatial econometric model, the spatial econometric models selected in this study are shown in Table 2.
4.1.2. Direct Effect and Spillover Effect. The spatial econometric modeling analysis can evaluate the complex spatial dependence between traffic zones. The influence of explanatory variables on the DiDi travel volume in the traffic zone itself and adjacent traffic zones not only includes the direct impact (direct effect) of explanatory variables in certain traffic subareas on the DiDi travel volume in the traffic zone but also changes the travel volume in the adjacent traffic zone (spillover effect) to a certain extent [29]. SDM model introduces weight variables (including explanatory variables and explained variables of other traffic zones) on the basis of independent variables to realize the measure of the above direct effect and spillover effect [28]. Further, the SDM model is described in the form of a vector:

$$
\begin{aligned}
\left(I_{n}-\rho W\right) y & =\beta X+\theta W X+\iota_{n} \alpha+\varepsilon, \\
y & =\sum_{r=1}^{k} S_{r}(W) X_{r}+V(W) \iota_{n} \alpha+V(W) \varepsilon,
\end{aligned}
$$

where $\rho$ is the lag coefficient of spatial variable, $l_{n}$ is the identity matrix, $\left(I_{n}-\rho W\right)^{-1}=V(W), \quad$ and $S(W)=V(W)\left(I_{n} \beta+\theta W\right)$. Matrix transformation is performed on (5), as shown in the following:

$$
\begin{aligned}
{\left[\begin{array}{c}
y_{1} \\
y_{2} \\
\vdots \\
y_{n}
\end{array}\right]=} & {\left[\begin{array}{cccc}
S_{r}(W)_{11} & S_{r}(W)_{12} & \cdots & S_{r}(W)_{1 n} \\
S_{r}(W)_{21} & S_{r}(W)_{22} & \cdots & S_{r}(W)_{2 n} \\
\vdots & \vdots & \ddots & \vdots \\
S_{r}(W)_{n 1} & S_{r}(W)_{n 2} & \cdots & S_{r}(W)_{n n}
\end{array}\right] \times\left[\begin{array}{c}
x_{1} \\
x_{2} \\
\vdots \\
x_{n}
\end{array}\right] } \\
& +V(W) \iota_{n} \alpha+V(W) \varepsilon .
\end{aligned}
$$

Through the diagonal analysis of (6), the average direct effect $\bar{M}(k)_{d}$, the average total effect $\bar{M}(k)_{t}$, and the average spillover effect $\bar{M}(k)_{i}$ are as follows:

$$
\begin{aligned}
& \bar{M}(k)_{d}=\frac{\operatorname{tr}\left(S_{r}(W)\right)}{n}, \\
& \bar{M}(k)_{t}=\frac{\iota_{n}{ }^{T} S_{r}(W) \iota_{n}}{n}, \\
& \bar{M}(k)_{i}=\bar{M}(k)_{t}-\bar{M}(k)_{d} .
\end{aligned}
$$

4.2. Spatial Heterogeneity Model Construction. The classical GWR model is often used to explain spatial heterogeneity, but there are two defects in selecting this model in this study. On the one hand, it is assumed that all parameters in the model are kept at the same scale by default, but this study should select different scales of bandwidth for different built environment variables, which is contrary to the setting principle of the GWR model. On the other hand, the GWR model overemphasizes that the local influence of variables is greater than the whole, and the influence degree of all variables varies with space. The MGWR model refines the bandwidth by allowing variables to have different spatial smoothing terms, which solves the limitation that all variables in GWR have the same bandwidth. At the same time, the specific bandwidth of each variable can also be used as a 


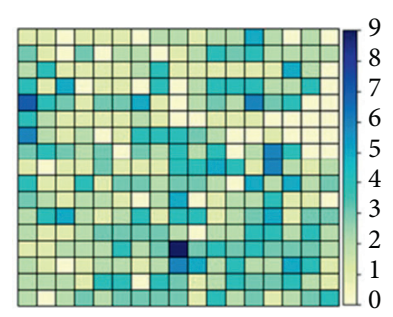

(a)

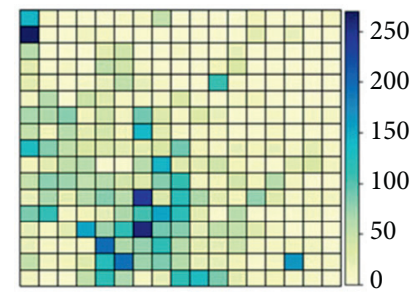

(e)

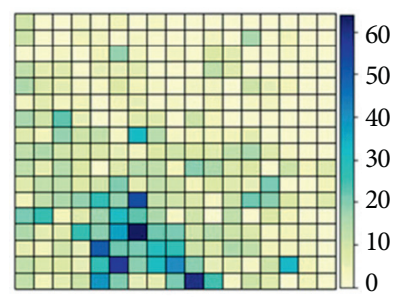

(i)

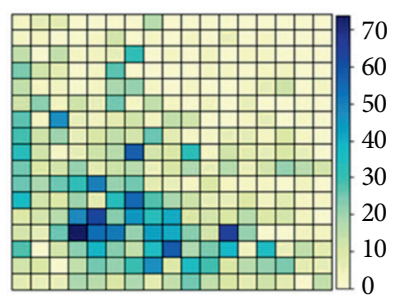

(m)

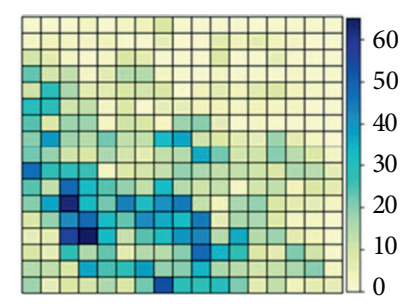

(b)

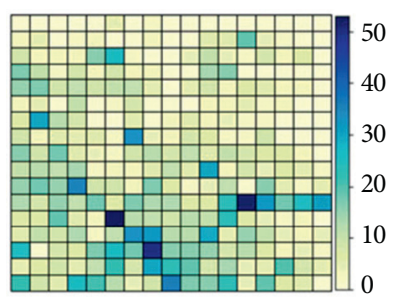

(f)

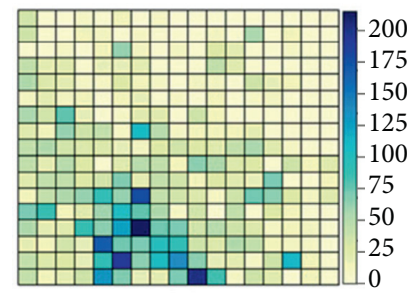

(j)

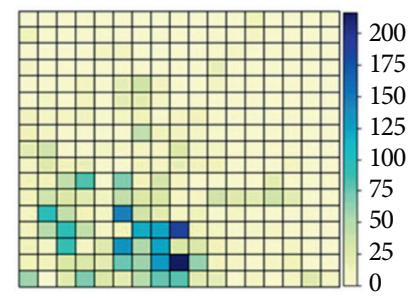

(n)

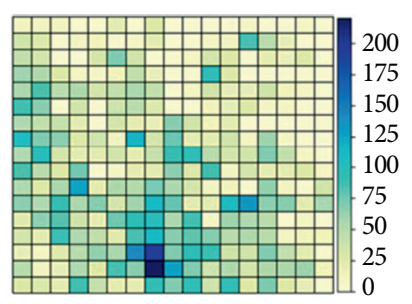

(c)

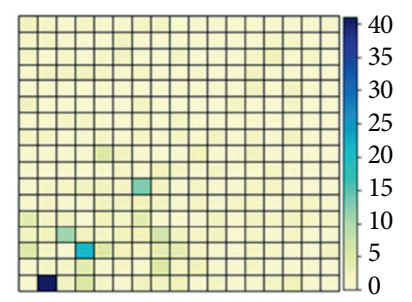

(g)

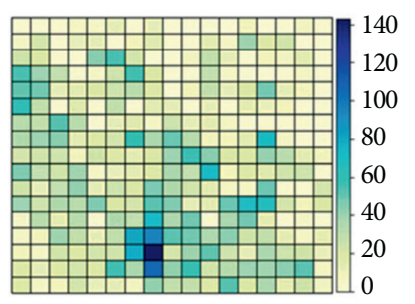

(k)

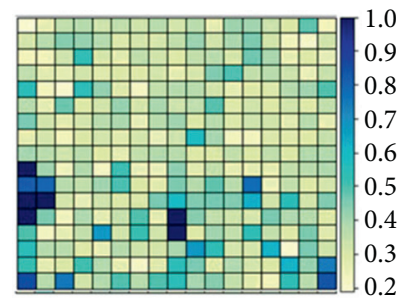

(o)

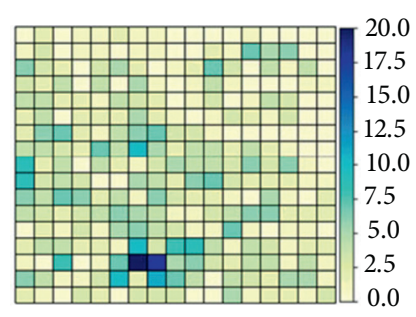

(d)

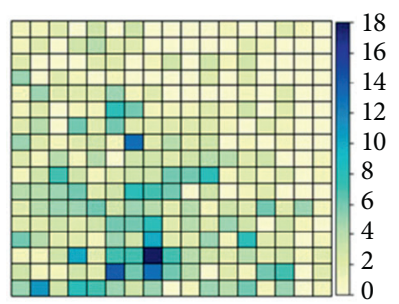

(h)

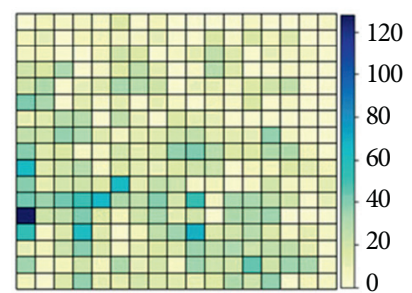

(1)

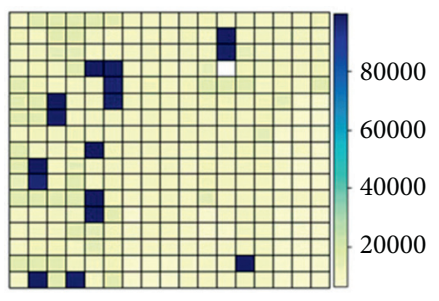

(p)

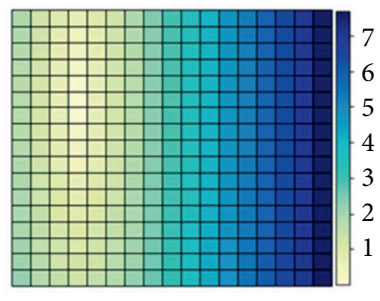

(q)

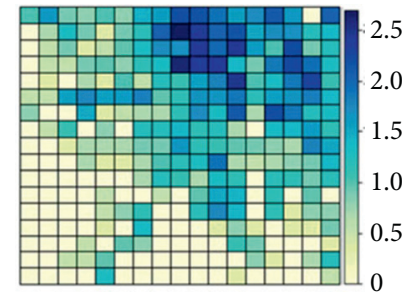

(r)

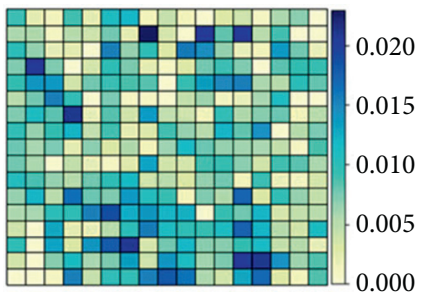

(s)

FIGURE 4: Heatmaps of independent variables distribution. (a) Bus station (BS). (b) Residential district (RD). (c) Catering service (CS). (d) Shopping service (SS). (e) Corporate business (CB). (f) Sports and leisure services (SL). (g) Science zone (SZ). (h) Public facility (PF). (i) Finance and insurance services (FI). (j) Education and culture (EC). (k) Life services (LS). (l) Medical and health (MH). (m) Government and administration (GA). (n) Accommodation service (AS). (o) Land-use mix (LM). (p) People density (PD). (q) Distance to CBD (DC). (r) Orientation order (OO). (s) Road network density (RD).

measure for each spatial process scale, which makes the spatial process model generated by multiple bandwidths more practical [33].

Based on the MGWR model, this study examined the spatial scale differences of online car-hailing travel factors under the influence of orientation-order indicator and road network density, respectively. The formula is as follows:

$$
y_{i}=\sum_{j=1}^{k} \beta_{b i j}\left(u_{i}, v_{i}\right) x_{i j}+\varepsilon_{i},
$$


TABLE 2: Spatial econometric model selection.

\begin{tabular}{|c|c|c|c|}
\hline Model & $\begin{array}{c}\text { Spatial } \\
\text { parameters }\end{array}$ & Basic form of the model & The influencing factors of travel volume in traffic zones \\
\hline OLS & $\lambda=\theta=\gamma=0$ & $Y_{i}=\beta X_{i}+\varepsilon$ & The built environment of the traffic zone \\
\hline SAR & $\theta=\gamma=0$ & $Y_{i}=\lambda \sum_{j=1}^{n} W_{i j} Y_{i}+\beta X_{i}+\varepsilon$ & $\begin{array}{c}\text { The built environment of the traffic zone and the } \mathrm{DiDi} \\
\text { travel volume in the adjacent traffic district }\end{array}$ \\
\hline SEM & $\lambda=\theta=0$ & $Y_{i}=\beta X_{i}+\gamma \sum_{j=1}^{n} W_{i j} \zeta+\varepsilon$ & $\begin{array}{c}\text { The spatial correlation between unobservable } \\
\text { independent variables }\end{array}$ \\
\hline SDM & $\gamma=0$ & $Y_{i}=\lambda \sum_{j=1}^{n} W_{i j} Y_{i}+\theta \sum_{j=1}^{n} W_{i j} X_{i}+\beta X_{i}+\varepsilon$ & $\begin{array}{l}\text { The built environment of the traffic zone, the DiDi } \\
\text { travel volume, and the built environment of the } \\
\text { adjacent traffic zone }\end{array}$ \\
\hline $\begin{array}{l}\text { SDEM (spatial } \\
\text { Durbin error } \\
\text { model) }\end{array}$ & $\lambda=0$ & $Y_{i}=\theta \sum_{j=1}^{n} W_{i j} X_{i}+\beta X_{i}+\sigma \sum_{j=1}^{n} W_{i j} \zeta+\varepsilon$ & $\begin{array}{c}\text { The correlation between independent variables of the } \\
\text { built environment and the spatial correlation of } \\
\text { unobservable variables }\end{array}$ \\
\hline
\end{tabular}

where $\beta_{b i j}$ represents the regression coefficient of the local variable, $b i j$ is the bandwidth used by the regression coefficient of the variable $j,\left(u_{i}, v_{i}\right)$ represents the spatial coordinates of the sample point, $x_{i j}$ is the observed value of the variable $j$ at the sample point $i$, and $\varepsilon_{i}$ is the random disturbance term. This study selected the bandwidth according to the Akaike information criterion (AIC) [34], which is widely used to measure the quality of the statistical model.

\section{Results and Discussion}

To verify the accuracy of the two factors of orientation order and road network density to characterize the neighborhood design under the data statistical condition of this study, the modeling and comparative analysis were carried out in three cases: in Case $1(\mathrm{RD})$, road network density was used to characterize neighborhood design as one of the independent variables; in Case 2 ( $\mathrm{RD}$ and $\mathrm{OO}$ ), both road network density and orientation-order indicator were put into the model as independent variables; in Case $3(\mathrm{OO})$, the orientation-order indicator was selected.

Before modeling the spatial econometric model, the distribution characteristics of spatial data should be investigated first. Moran's I was applied to test whether there is a correlation between the same independent variables in different traffic zones, and then Moran's I values were obtained as shown in Table 3. From the analysis in Table 3, according to $p$ value, the global Moran index values of all variables are significant (except population density); that is, there is a significant spatial correlation between adjacent traffic zones.

5.1. Optimal Model Selection. Through models comparison, this section selects the best fitting model from spatial correlation and heterogeneity.

5.1.1. Spatial Correlation Optimal Model Selection. The influence analysis of spatial correlation needs to select an appropriate spatial econometric model. Previous studies mostly relied on the Lagrange multiplier test and other methods. In this study, regression analysis is carried out for each model, and the optimal model is selected according to the judgment criterion that the greater the judgment
TABle 3: Moran's index value of the variable.

\begin{tabular}{lcc}
\hline Variable & Moran's I & $z$-statistic \\
\hline Bus station & 0.191 & $7.700^{*}$ \\
Residential district & 0.693 & $24.572^{* * *}$ \\
Catering service & 0.476 & $17.538^{* * *}$ \\
Shopping service & 0.294 & $11.665^{* *}$ \\
Corporate business & 0.534 & $19.414^{* * *}$ \\
Sports and leisure service & 0.445 & $16.538^{* * *}$ \\
Science zone & 0.203 & $8.718^{* *}$ \\
Public facility & 0.350 & $13.462^{* * *}$ \\
Finance and insurance services & 0.546 & $19.803^{* * *}$ \\
Education and culture & 0.575 & $21.747^{* * *}$ \\
Life service & 0.402 & $15.139^{* * *}$ \\
Medical and health & 0.366 & $13.968^{* * *}$ \\
Government and administration & 0.575 & $21.754^{* * *}$ \\
Accommodation service & 0.655 & $23.332^{* * *}$ \\
Land-use mix & 0.256 & $10.432^{* *}$ \\
Population density & 0.052 & 2.827 \\
Distance to CBD & 0.561 & $21.300^{* * *}$ \\
Orientation order (road network & 0.203 & $8.167^{*}$ \\
density) & $(0.148)$ & $(4.917)$ \\
\hline
\end{tabular}

Note: ${ }^{* * *},{ }^{* *}$, and ${ }^{*}$ refer to significance at the level of $0.01,0.05$, and 0.1 , respectively.

coefficient (adjusted $R^{2}$ ) and log-likelihood are, the smaller the AIC is. The model results of the peak period (13:00 to 14: 00) are shown in Table 4.

Table 4 shows that the fitting results of SDM models are significantly better than other models, and the descending order of fit goodness order of the SDM model in three cases is shown as Case 3, Case 2, and Case 1. Specifically speaking, in Case 1 , the adjusted $R^{2}$ of the SDM model is 0.725 , which is 0.087 better than that in Case 1 (0.712), and log-likelihood and AIC ( -3265.376 and 6867.753) increased and decreased to some extent, respectively, compared with Case 1 $\left(-3312.376\right.$ and 6972.307). In Case 3 , the adjusted $R^{2}$ of the SDM model is 0.774 , an improvement of 0.062 compared with Case 1, and log-likelihood and AIC (-3265.376 and 6867.753) also increased and decreased to a certain extent separately compared with Case 1 . From the above analysis results, the orientation-order indicator does have a certain impact on the online car-hailing travel behavior, and the fitting degree of the single consideration of the orientationorder indicator (Case3) is higher than that of the model 
TABLE 4: Comparison of fitting results of OLS, SAR, SDM, and SDEM models.

\begin{tabular}{|c|c|c|c|c|c|c|}
\hline \multicolumn{2}{|c|}{ Parameters } & OLS & SAR & SEM & SDM & SDEM \\
\hline \multirow{3}{*}{ Case1 (RD) } & Adjusted $R^{2}$ & 0.672 & 0.689 & 0.627 & 0.712 & 0.705 \\
\hline & Log-likelihood & - & -3523.548 & -6933.912 & -3312.376 & -3465.321 \\
\hline & AIC & - & 7085.096 & 7892.849 & 6972.307 & 6984.574 \\
\hline \multirow{3}{*}{ Case2 (RD and $\mathrm{OO})$} & Adjusted R2 & 0.683 & 0.695 & 0.679 & 0.725 & 0.710 \\
\hline & Log-likelihood & - & -3494.656 & -6859.320 & -3346.114 & -3462.224 \\
\hline & AIC & - & 7085.321 & 7796.172 & 6873.569 & 6919.321 \\
\hline \multirow{3}{*}{ Case3 (OO) } & Adjusted $R^{2}$ & 0.699 & 0.704 & 0.686 & 0.774 & 0.716 \\
\hline & Log-likelihood & - & -3473.016 & -6782.561 & -3265.376 & -3461.246 \\
\hline & AIC & - & 7086.033 & 7782.753 & 6867.753 & 6883.154 \\
\hline
\end{tabular}

considering both the orientation-order indicator and road network density (Case2). It indicates that road network density is not the key influencing factor of online car-hailing travel behavior in this study, and more attention should be paid to the orientation-order indicator of neighborhood and road network connectivity. In other words, the higher the road grid is, the better its connectivity will be, thus attracting more DiDi travel volume.

5.1.2. Spatial Heterogeneity Optimal Model Selection. In this part, GWR and MGWR models are, respectively, used for comparative analysis of spatial heterogeneity, and the optimal bandwidth (judged according to AICc value, and adaptive bandwidth is selected) and spatial kernel function (Gaussian kernel function with universality is selected) are used for modeling analysis [35]. Table 5 implies the comparison results of the GWR and MGWR model of the boarding behavior of DiDi vehicles in the peak period.

From the analysis of Table 5, the fitting results of the MGWR models are better than the GWR model. And the descending order of fit goodness of the SDM model in three cases is shown as Case 3, Case 2, and Case 1. In detail, the adjusted $R^{2}$ of the MGWR model in Case 2 is 0.797 , which is 0.028 improved compared with Case 1, and the AICc and RSS (431.561 and 53.976) are both reduced to a certain extent compared with Case 1 (432.487 and 54.385). In Case 3, the adjusted $R^{2}$ of the MGWR model is 0.826 , which is 0.057 higher than that in Case 1, and the AICc and RSS (429.567 and 51.245) are reduced to a certain extent compared with Case 1. Based on the above analysis results, we can get the conclusion consistent with the optimal spatial correlation model; that is, the fitting result of the model (Case 3), which only considers the orientation-order indicator between the road network density and the orientation-order indicator, is the best. From the perspective of spatial heterogeneity, this study also verifies that the orientation-order indicator has a certain influence on online car-hailing travel behavior and further indicates that it is reasonable to consider the orientation-order indicator as one of the representative factors of neighborhood design.

5.2. Optimal Model Estimation. According to the selection of optimal model in Section 5.1, the SDM model and MGWR model of Case 3 (selecting the orientation-order indicator as one of the independent variables) have the highest fitting
TABLE 5: Comparative analysis of the evaluation results of GWR and MGWR models.

\begin{tabular}{lccc}
\hline & Variable & GWR & MGWR \\
\hline & $R^{2}$ & 0.763 & 0.803 \\
Case1 (RD) & Adjusted $R^{2}$ & 0.715 & 0.769 \\
& AICc & 481.569 & 432.487 \\
& RSS (residual sum of the & 68.420 & 54.385 \\
\hline \multirow{4}{*}{ square) } & & \\
Case2 (RD and & $R^{2}$ & 0.781 & 0.823 \\
OO) & Adjusted $R^{2}$ & 0.749 & 0.797 \\
& AICc & 478.910 & 431.561 \\
& RSS & 64.013 & 53.976 \\
Case3 (OO) & $R^{2}$ & 0.767 & 0.861 \\
& Adjusted $R^{2}$ & 0.735 & 0.826 \\
& AICc & 480.518 & 429.567 \\
& RSS & 67.289 & 51.245 \\
\hline
\end{tabular}

degree. Therefore, this part estimates and analyzes the spatial correlation and spatial heterogeneity based on the optimal model so as to quantitatively analyze the impact of each variable on the built environment, especially the impact of the orientation-order indicator.

\subsubsection{Spatial Correlation Analysis-SDM Model Estimation.}

The model parameter estimation results of the peak period obtained based on the SDM model are shown in Table 6. It can be seen that the bus station POI, residential district POI, catering service POI, shopping service POI, corporate business, land-use mix, and orientation order have significant positive correlations with the generation of DiDi travel volume. That is, the greater the development intensity of the traffic zone, the greater the intensity of people's travel activities and the higher the demand for DiDi travel. The larger the orientation-order indicator is, the higher the grid orientation of the city streets is, and the streets will be extended orderly in all orientations, which is generally reflected in the city center with the relatively perfect road network, so it will attract more traffic volume of online car-hailing.

Specifically, the catering service POI, corporate business POI, and land-use mix in the surrounding traffic community have significant positive effects on the DiDi travel volume of this traffic zone. However, sports and leisure service POI and orientation order of the surrounding traffic community have noteworthy negative influences on the traffic volume of this traffic zone. The spatial lag variable coefficient $\rho$ is positive 
TABLE 6: Comparison of parameter estimation results of SDM model.

\begin{tabular}{lcc}
\hline Variable & Coefficient & $t$-statistic \\
\hline Constant & $6.202^{* * *}$ & 4.360 \\
Bus station (BS) & $0.146^{*}$ & 2.252 \\
Residential district (RD) & $0.201^{* * *}$ & 3.612 \\
Catering service (CS) & $0.174^{*}$ & 2.156 \\
Shopping service (SS) & $0.131^{*}$ & 2.218 \\
Corporate business (CB) & $0.091^{* *}$ & 2.141 \\
Sports and leisure service (SL) & 0.009 & 0.135 \\
Science zone (SZ) & 0.059 & 0.761 \\
Public facility (PF) & -0.027 & -0.466 \\
Finance and insurance services (FI) & -0.009 & -0.141 \\
Education and culture (EC) & 0.065 & 1.274 \\
Life service (LS) & $0.140^{*}$ & 2.162 \\
Medical and health (MH) & 0.016 & 0.283 \\
Government and administration (GA) & -0.023 & -0.735 \\
Accommodation service (AS) & 0.053 & 1.075 \\
Land-use mix (LM) & $0.218^{*}$ & 0.951 \\
Population density (PD) & -0.023 & -0.761 \\
Distance to CBD (DC) & 0.034 & 1.235 \\
Orientation order (OO) & $0.147^{*}$ & 1.847 \\
$W *$ BS & 0.101 & 0.540 \\
$W *$ RD & $-0.092^{*}$ & -0.651 \\
$W *$ CS & $0.405^{* * *}$ & 2.619 \\
$W *$ SS & 0.111 & 0.753 \\
$W *$ CB & $0.171^{*}$ & 1.760 \\
$W *$ SL & $-0.403^{* * *}$ & -2.923 \\
$W *$ SZ & 0.051 & 0.297 \\
$W *$ PF & -0.099 & -0.807 \\
$W *$ FI & $-0.218^{*}$ & -1.687 \\
$W *$ EC & 0.147 & 1.262 \\
$W *$ LI & -0.246 & -1.463 \\
$W *$ MH & 0.060 & 0.463 \\
$W *$ GA & -0.010 & -0.091 \\
$W *$ AS & -0.008 & -0.080 \\
$W *$ LM & $-1.442^{* *}$ & -2.259 \\
$W *$ PD & -0.479 & -1.593 \\
$W *$ DC & -0.185 & -1.046 \\
$W *$ OO & $-0.095^{* *}$ & -2.065 \\
$\rho$ & $0.111^{* * *}$ & 7.139 \\
\hline
\end{tabular}

Note: ${ }^{* *},{ }^{* *}$, and ${ }^{*}$ refer to significance at the level of $0.01,0.05$, and 0.1 , respectively; $W$ is the spatial weight matrix.

and significant, and the traffic zone has a significant spillover effect on the adjacent traffic zones. Therefore, it is necessary to further test and calibrate the size of the spillover effect so as to reveal its spatial correlation impact on DiDi travel behavior. The analysis of direct effect, spillover effect, and total effect is shown in Table 7.

From the direct effect, it can be seen that the direct effects of the bus station, residential district, catering service, shopping service, corporate business, life service, and orientation order are all positive and significant; that is, the increase of these independent variables in a certain traffic zone will improve the volume of DiDi travel in the traffic zone.

Through the analysis of the spillover effect, the spillover effect of catering service, corporate business, and orientation order is positive. Specifically, the spillover effect of the first two variables is positive. A possible explanation is that the number of people having meals and going to and from companies in a certain traffic zone increases in the noon peak, which would drive the increase of DiDi travel demand in its surrounding traffic zones; the orientation order illustrates that the greater the value of the orientation order in this traffic zone, the greater the degree of uniform distribution of streets in all orientations and the higher the attraction value of the traffic zone to $\mathrm{DiDi}$ travel volume. Due to the market effect, the travel demand of the surrounding traffic zones would boost to a certain extent. However, the spillover effects of sports leisure service and land-use mix are negative. To be more specific, the increase of sports leisure service in a certain traffic area would not improve the DiDi travel demand of surrounding traffic zones, which may demonstrate that the sports leisure and entertainment facilities in the area are relatively sound, thus attracting a large number of DiDi trips, and then the demand of the adjacent communities could decrease correspondingly; the greater the land-use mix in a traffic zone, the stronger the intensity of travel activities in the region, which would undoubtedly reduce the travel demand of surrounding traffic zones.

From the total effect, it could be inferred that bus station, catering service, shopping service, corporate business, education and culture, and orientation order have noteworthy total effects on DiDi travel demand. Through the above analysis, the rationality of using the orientation-order indicator to characterize neighborhood design is demonstrated from the perspective of spatial correlation.

\subsubsection{Spatial Heterogeneity Analysis-MGWR Model} Estimation. The spatial distribution of significant independent variable coefficients during the peak period (13: 00 to 14:00) calculated by the MGWR model, namely, the influencing mechanism of spatial heterogeneity, is visually analyzed, as indicated in Figure 5. The analysis of significant variables is as follows: as shown in Figure 5(a), the spatial distribution of bus station POI coefficient displays a wavy decreasing trend from northeast to southwest. This illustrates that the impact of bus station POI on DiDi travel is conspicuous in the suburbs, especially in the northeast outside the Second Ring Road; that is, DiDi travel is always generated near the bus station in this region. Therefore, it is speculated that the flexibility and convenience of DiDi vehicles may make it a supplement to bus travel, that is, the formation of "DiDi travel + Bus" travel mode. However, this travel mode is not obvious in the Second Ring Road, which may be due to the more convenient public transportation system in the city center. This finding is contrary to the conclusion of Tu et al. [36], which indicates that taxi travel does not supplement bus travel and may be related to the low income of some bus passengers. Although taxis and online car-hailing have different travel behaviors, their service modes are to provide door-to-door service for the public. The above two research studies are contradictory, perhaps because of the different study areas (one is in the United States; another is in China). Therefore, the accuracy of this inference should be verified additionally in future studies. 
TABle 7: Direct effect, spillover effect, and total effect of SDM model.

\begin{tabular}{|c|c|c|c|c|c|c|}
\hline \multirow{2}{*}{ Variable } & \multicolumn{2}{|c|}{ Direct effect } & \multicolumn{2}{|c|}{ Spillover effect } & \multicolumn{2}{|c|}{ Total effect } \\
\hline & Coefficient & $t$-statistic & Coefficient & $t$-statistic & Coefficient & $t$-statistic \\
\hline Bus station & $0.147^{* *}$ & 2.264 & 0.060 & 0.352 & $0.207^{* * *}$ & 1.130 \\
\hline Residential district & $0.205^{* * *}$ & 4.508 & -0.139 & -0.825 & 0.066 & 0.387 \\
\hline Catering service & $0.190^{* *}$ & 3.250 & $0.548^{* * *}$ & 5.930 & $0.738^{* * *}$ & 3.915 \\
\hline Shopping service & $0.137^{* *}$ & 1.965 & 0.034 & 0.195 & $0.171^{* *}$ & 1.865 \\
\hline Corporate business & $0.032^{*}$ & 1.606 & $0.257^{* *}$ & 2.350 & $0.289^{* * *}$ & 2.829 \\
\hline Sports and leisure service & 0.002 & 0.026 & $-0.532^{* * *}$ & -3.680 & $-0.530^{* * *}$ & -3.285 \\
\hline Science zone & 0.061 & 0.824 & -0.039 & -0.198 & 0.022 & 0.108 \\
\hline Public facility & -0.039 & -0.801 & -0.106 & -0.708 & -0.145 & -0.886 \\
\hline Finance and insurance services & -0.018 & -0.349 & -0.211 & -1.383 & -0.229 & -1.538 \\
\hline Education and culture & 0.071 & 1.380 & 0.179 & 1.325 & $0.250^{*}$ & 1.884 \\
\hline Life service & $0.115^{*}$ & 1.904 & -0.181 & -0.877 & -0.066 & -0.202 \\
\hline Medical and health & 0.014 & 0.237 & 0.063 & 0.432 & 0.077 & 0.538 \\
\hline Government and administration & -0.031 & -0.960 & -0.023 & -0.193 & -0.054 & -0.433 \\
\hline Accommodation service & 0.057 & 1.136 & -0.009 & -0.079 & 0.048 & 0.501 \\
\hline Land-use mix & 0.014 & 1.054 & $-0.233^{* *}$ & 2.512 & $-0.219^{* * *}$ & -3.298 \\
\hline Population density & -0.032 & -1.002 & -0.503 & -1.528 & -0.535 & -1.478 \\
\hline Distance to CBD & 0.030 & 1.728 & -0.200 & -1.426 & -0.170 & -1.383 \\
\hline Orientation order & $0.034^{*}$ & 1.747 & $0.131^{* *}$ & 1.689 & $0.165^{* *}$ & 1.733 \\
\hline
\end{tabular}

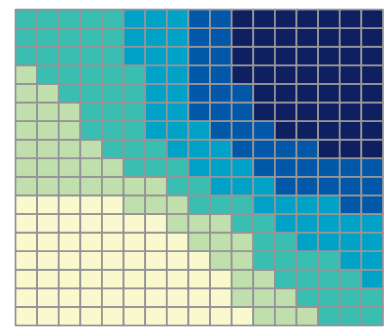

$$
\begin{array}{ll}
-0.05,-0.01 & \bullet 0.07,0.12 \\
-0.01,0.03 & \bullet 0.12,0.17 \\
0.03,0.07 & \bullet 0.17,0.22
\end{array}
$$

(a)

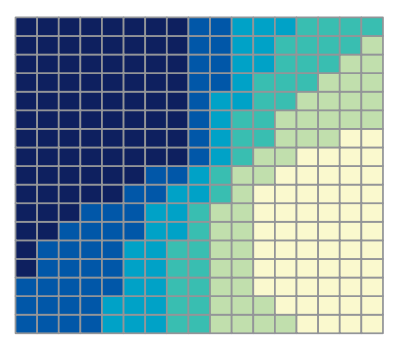

$0.07,0.07$

$0.07,0.07$

- $0.07,0.08$
$0.08,0.08$

- $0.08,0.08$

- $0.08,0.08$

(e)

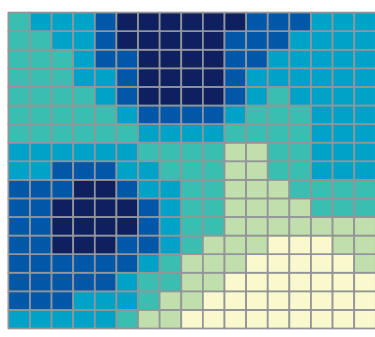

$-0.09,-0.05 \bullet 0.03,0.07$

$-0.05,-0.05 \bullet 0.07,0.11$

$-0.01,0.03$ • $0.11,0.27$

(b)

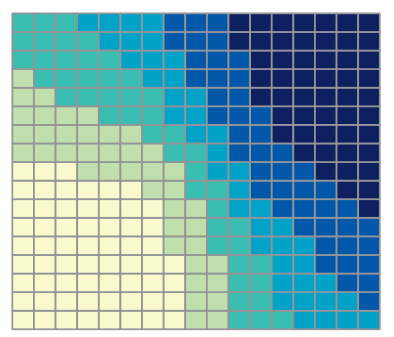

\begin{abstract}
$0.05,0.06$
$0.06,0.08$

- $0.08,0.09$

$0.09,0.10$

- $0.10,0.11$

- $0.11,0.13$
\end{abstract}

(f)

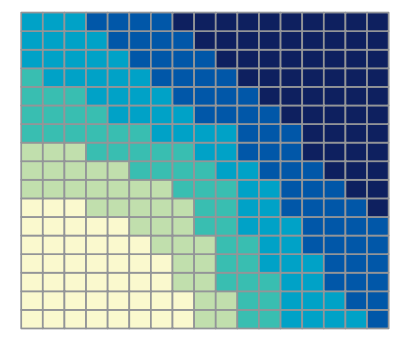

- $0.15,0.16$

- $0.16,0.16$

- $0.16,0.17$

(c)

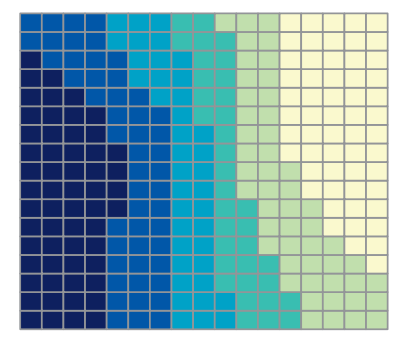

$-0.01,0.00 \bullet 0.02,0.04$

$0.01,0.01 \bullet 0.04,0.05$

- $0.01,0.02$

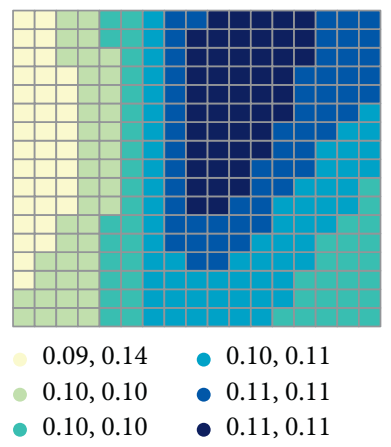

(d)

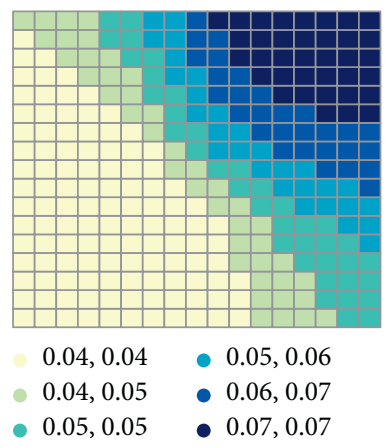

(h)

FIGURE 5: Spatial distribution of coefficients of significant variables in case3. (a) Bus station (BS). (b) Residential district (RD). (c) Catering service (CS). (d) Sports and leisure services (SL). (e) Corporate business (CB). (f) Life services (LS). (g) Land-use mix (LM). (h) Orientation order $(\mathrm{OO})$.

As shown in Figure 5(b), the residential district POI has a powerful positive impact on DiDi travel, and the impact is significant in the north and southwest corners of the region. The residential district POI is relatively dense in the southwest direction (Figure 4(b)), which is consistent with the impact intensity; however, the residential district POI is relatively sparse in the northern area (Figure 4(b)). The reason may be that the land-use types outside the Second Ring Road are relatively single and less abundant than those in the urban area, mainly for residential and undeveloped land use, and the DiDi travel mainly provides convenient services for residents in residential areas, so it shows a strong 
influence here. Although this finding is consistent with the conclusion of Zhuo et al. [37], that is, the contribution of residential density to taxi travel is positive, it suggests that residential density does not have spatial heterogeneity, which may be due to the situations of the different effects in different zones hidden in the study.

What is confusing is that, in Figure 5(c), the coefficient spatial distribution of catering service POI displays a wavy decreasing trend from northeast to southwest. It may be because the northeast of the study area is far away from the city center, and the public transportation is not very developed. Thus, most passengers choose $\mathrm{DiDi}$ vehicles to travel for meals here, while the city center is the opposite. The spatial distribution trend of coefficient of life service POI (as shown in Figure 5(f)) is similar to that of catering service POI.

The area where DiDi travel is most affected by shopping service POI is not the southwest region but the northern and north-central region (see Figure 5(d)). The southwest region (near Chunxi Road) does generate a large DiDi travel volume, but the region's highly diverse land-use mix may be more than just attracted by shops. The shops in the northern part of the region are mainly concentrated around the residential district, which may be the reason why the shops have a greater influence in this area.

As can be seen from Figure 5(e), the coefficient of corporate business POI decreases from the northwest corner to the southeast corner, while the POI distribution is more in the southwest city center (Figure 4(e)). Especially in the southwest region, the density distribution of corporate business POI is relatively large, but the spatial impact intensity is small, which may be owing to the public transportation in the city center being more developed, and people often choose public transportation in the process of commuting. In addition, corporate business POI is densely distributed in a few areas of the northwest corner, which is also easy to explain. It indicates that there are some companies in the northwest corner because the bus system is relatively underdeveloped and people often use $\mathrm{DiDi}$ to achieve commuting.

It can be seen from Figure 5(g) that the land-use mix has a strong positive impact on online car-hailing travel, especially in the west; that is, areas with high land-use mix have more online car-hailing travel. However, this finding is different from studies by some researchers who believe that land-use mix is an effective strategy to reduce car travel by integrating adequate living facilities (such as office space, residential areas, shops, and other facilities) [38]. This study area near Chunxi Road is a famous business district in Chengdu, with a high level of land-use combination and numerous buildings such as shopping, entertainment, office, residence, and hospital. According to the research of Kockelman [39], in areas with a high land-use mix, the vehicle mileage of nonworking travel is less. However, the volume of DiDi travel is higher in this area. One possible explanation is that regions with high diversity are more attractive than other regions; that is, due to lower travel costs, it improves accessibility to multiple destinations and increases nonworking travel [40].
As shown in Figure 5(h), the orientation order has a strong positive influence on DiDi travel, and its change trend gradually decreases from the northeast corner to the southwest corner. Among them, the $\varphi$ value in the northeast direction of the study area is large, indicating that the street orientation in this area is in good order, with a higher node degree, fewer dead-ends, and smaller circuity. At this time, the streets are evenly distributed in each orientation [15], which is in line with the structural form of the radial road network in Chengdu, and the street structure would increase the demand for DiDi travel accordingly. Moreover, the $\varphi$ value is small in the southwest region. As the center of the radial road network, the crisscross road network leads to poor orientation order, and people tend to rely on the public transport system to travel. It can be seen that this indicator can well highlight the impact of neighborhood design on travel behavior, which is also consistent with the spatial correlation research results mentioned previously.

\section{Conclusion}

In this work, the road network orientation entropy indicator was introduced to analyze the spatiotemporal impact of the urban built environment on DiDi travel from two perspectives of spatial correlation and spatial heterogeneity. The main conclusions are as follows.

In this study, the orientation-order indicator was proposed as one of the characterization factors of the neighborhood design dimension in the "five Ds." Through modeling and analysis in three cases (considering road network density, considering road network density and orientation-order indicator, and considering orientationorder indicator). It is concluded that the orientation-order indicator does have a certain influence on online car-hailing travel behavior. And the fitting degree of the model considering the single orientation-order indicator is higher than that considering both the orientation-order indicator and road network density. This indicates that road network density is not the main influencing factor of DiDi travel behavior in the case of this study, and more attention should be paid to the orientation order of neighborhoods and road network connectivity. That is, the greater the orientationorder indicator, the higher the grided degree of the road and the better its connectivity, thus attracting more DiDi trips. The possible reasons for the above results are that the highly grided road network will make it easier for car-hailing companies to operate efficiently, making their services more convenient and attractive or making it easier for customers and drivers to meet up and get on or off. And it may also be that the higher the connectivity of the road network, the lower the travel cost, which will attract more vehicle trips, and because of the convenience of online car-hailing, it is easier for travelers to choose. Moreover, through spatial correlation and heterogeneity analysis, the significant impact on DiDi travel behavior was further quantified. At the same time, the above conclusions also verify that it is reasonable and necessary to consider the orientation-order indicator as to the constituent elements of the urban built environment. 
From the perspectives of spatial correlation and heterogeneity analysis, this study constructed spatial econometric models, respectively, and screened out the best fitting models SDM and MGWR. Based on the estimation results of the SDM model, the direct effect, spillover effect, and total effect are quantified. The results show that it is necessary to include the spatial spillover effect in the study of the impact of the built environment on online car-hailing demand based on the traffic zoning scale. The built environment of the traffic zone not only significantly affects the demand for online car-hailing wherein itself but also has significant impacts on the demand for ride-hailing in other traffic zones. Specifically, catering service, corporate business, and orientation-order indicators have significant positive impacts, while sports and leisure service and land-use mix have vital negative impacts. Based on the estimation results of the MGWR model, bus station, residential district, catering service, shopping service, corporate business, land-use mix, and orientation-order indicators are the main influencing factors of DiDi travel from the perspective of spatial variation coefficient analysis, among which residential district and orientation order have remarkable positive impacts.

This study analyzed the influence of various factors on DiDi travel, which can effectively help DiDi company to operate more effectively, upgrade the dynamic ridematching algorithm, then improve the order receiving efficiency of drivers, and reduce the waiting time or detour time. DiDi travel may be a complementary mode for public transport, especially in the eastern region outside the Second Ring Road, and the development of this mode echoes the development of the interconnected multimodal transport system. Population density, sports and leisure service, scenic zone, public facility, medical and health service, government and accommodation, and other POIs have no significant impact on online car-hailing travel, and these variables have been proved to be important in other pieces of literature, indicating that perhaps when selecting appropriate variables, measures should be taken according to local conditions.

This study shows that the larger the orientation-order indicator has a significant impact on online car-hailing travel, implying that orientation order leads to many online car-hailing trips per capita or a high share of online carhailing mode. At the same time, when the orientation order is high, it is possible to have a significant impact on the overall vehicle travel demand of the city, rather than just on online car-hailing. Therefore, travel data of other travel modes can be collected for verification in the future. In addition, the choice of travel behavior factors can be expanded in future research, including travel time, travel distance, and land-use mix characteristics of travel destinations, to reveal the close relationship between the built environment and DiDi travel.

\section{Data Availability}

The data used to support the findings of this study are available from the Gaia Open Dataset by DiDi. They can be obtained through the following website: https://outreach. didichuxing.com/research/opendata/.

\section{Conflicts of Interest}

The authors declare that there are no conflicts of interest regarding the publication of this paper.

\section{Acknowledgments}

This work was funded by the National Key Research and Development Program of China (Grant no. 2019YFB1600500) and the Projects Supported by Special Funds for Urban Construction (Grant no. SZJJ2019-22). The authors acknowledge the Gaia Open Dataset by DiDi for the support in data collection.

\section{References}

[1] S. L. Handy, M. G. Boarnet, R. Ewing, and R. E. Killingsworth, "How the built environment affects physical activity," American Journal of Preventive Medicine, vol. 23, no. 2, pp. 64-73, 2002.

[2] R. Ewing and R. Cervero, "Travel and the built environment," Journal of the American Planning Association, vol. 76, no. 3, pp. 265-294, 2010.

[3] T. Li, P. Jing, L. C. Li, D. Sun, and W. Yan, "Revealing the varying impact of urban built environment on online carhailing travel in spatio-temporal dimension: an exploratory analysis in Chengdu, China," Sustainability, vol. 11, no. 5, 2019.

[4] F. Alemi, G. Circella, S. Handy, and P. Mokhtarian, "What influences travelers to use Uber? Exploring the factors affecting the adoption of on-demand ride services in California," Travel Behaviour and Society, vol. 13, pp. 88-104, 2018.

[5] H. Yu and Z.-R. Peng, "Exploring the spatial variation of ridesourcing demand and its relationship to built environment and socioeconomic factors with the geographically weighted Poisson regression," Journal of Transport Geography, vol. 75, no. 2, pp. 147-163, 2019.

[6] H. Yu and Z.-R. Peng, "The impacts of built environment on ridesourcing demand: a neighbourhood level analysis in Austin, Texas," Urban Studies, vol. 57, no. 1, pp. 152-175, 2020.

[7] S. Sabouri, K. Park, A. Smith, G. Tian, and R. Ewing, "Exploring the influence of built environment on Uber demand," Transportation Research Part D: Transport and Environment, vol. 81, Article ID 102296, 2020.

[8] M. Tu, W. X. Li, O. Orfila, Y. Li, and D. Gruyer, "Exploring nonlinear effects of the built environment on ridesplitting: evidence from Chengdu," Transportation Research Part D: Transport and Environment, vol. 93, Article ID 102776, 15 pages, 2021.

[9] W. Li, Z. Pu, Y. Li, and X. Ban, "Characterization of ridesplitting based on observed data: a case study of Chengdu, China," Transportation Research Part C: Emerging Technologies, vol. 100, pp. 330-353, 2019.

[10] S. Zhong, Z. Wang, Q. Wang, A. Liu, and J. Cui, "Exploring the spatially heterogeneous effects of urban built environment on road travel time variability," Journal of Transportation Engineering Part A Systems, vol. 82, no. 1, 2021.

[11] K. Choi, "The influence of the built environment on household vehicle travel by the urban typology in Calgary, Canada," Cities, vol. 75, no. 5, pp. 101-110, 2018. 
[12] Q. Liu, C. Ding, and P. Chen, "A panel analysis of the effect of the urban environment on the spatiotemporal pattern of taxi demand," Travel Behaviour and Society, vol. 18, no. 1, pp. 29-36, 2020.

[13] N. Mohajeri and A. Gudmundsson, "The evolution and complexity of urban street networks," Geographical Analysis, vol. 46, no. 4, pp. 345-367, 2014.

[14] S. A. Shaheen, N. D. Chan, and T. Gaynor, "Casual carpooling in the San Francisco Bay Area: understanding user characteristics, behaviors, and motivations," Transport Policy, vol. 51, pp. 165-173, 2016.

[15] Y. Wang, S. Wang, J. Wang, J. Wei, and C. Wang, "An empirical study of consumers' intention to use ride-sharing services: using an extended technology acceptance model," Transportation, vol. 47, no. 1, pp. 397-415, 2020.

[16] X. Tang, Z. Qin, F. Zhang et al., “A deep value-network based approach for multi-driver order dispatching," in Proceedings of the 25th ACM SIGKDD International Conference on Knowledge Discovery \& Data Mining, Anchorage, AK, USA, July 2019.

[17] M. Tu, Y. Li, W. Li, M. Tu, O. Orfila, and D. Gruyer, "Improving ridesplitting services using optimization procedures on a shareability network: a case study of Chengdu," Technological Forecasting and Social Change, vol. 149, Article ID 119733, 9 pages, 2019.

[18] L. David and E. Ahmed, "The minimum circuity Frontier and the journey to work," Regional Science and Urban Economics, vol. 39, no. 6, pp. 732-738, 2009.

[19] P. Parthasarathi, H. Hochmair, and D. Levinson, "Street network structure and household activity spaces," Urban Studies, vol. 52, no. 6, pp. 1090-1112, 2015.

[20] P. J. Lamíquiz and J. López-Domínguez, "Effects of built environment on walking at the neighbourhood scale. A new role for street networks by modelling their configurational accessibility?" Transportation Research, Part A:Policy and Practice, vol. 74, pp. 148-163, 2015.

[21] W. J. Li, D. Hu, and Y. Liu, "An improved measuring method for the information entropy of network topology," Transactions in GIS, vol. 22, pp. 1-17, 2018.

[22] M. Barthelemy, P. Bordin, H. Berestycki, and M Gribaudi, "Self-organization versus top-down planning in the evolution of a city," Scientific Reports, vol. 3, no. 1, p. 2153, 2013.

[23] A. Gudmundsson and N. Mohajeri, "Entropy and order in urban street networks," Scientific Reports, vol. 3, p. 3324, 2013.

[24] G. Boeing, "Urban spatial order: street network orientation, configuration, and entropy," Applied Network Science, vol. 4, no. 1, p. 19, 2019.

[25] J. Holtzclaw, R. Clear, H. Dittmar, D. Goldstein, and P. Haas, "Location efficiency: neighborhood and Socio-Economic characteristics determine Auto Ownership and Use - studies in Chicago, Los Angeles and san Francisco," Transportation Planning and Technology, vol. 25, no. 1, pp. 1-27, 2002.

[26] C. F. Choudhury, Y. Lang, J. D. A. E. Silva, and M. Ben-Akiva, "Modelling preferences for smart modes and services: a case study in Lisbon," Transportation Research Part A: Policy and Practice, vol. 115, no. 9, pp. 15-31, 2017.

[27] Y. Chen, M. Zhu, Q. Zhou, and Y. Qiao, "Research on spatiotemporal differentiation and influence mechanism of urban resilience in China based on mgwr model," International Journal of Environmental Research and Public Health, vol. 18, no. 3, p. 27, 2021.

[28] J. P. Lesage and M. M. Fischer, "Spatial growth regressions: model specification, estimation and interpretation," Spatial Economic Analysis, vol. 3, no. 3, pp. 275-304, 2007.
[29] Q. L. Li, Y. R. Lu, C. Ding, and X.-L. Ma, "Analysis of the impact of built environment on taxi travel demand considering spatial spillover effect," Journal of Transportation Systems Engineering and Information Technology, vol. 16, no. 5, pp. 39-44, 2016.

[30] C. H. Wang and N. Chen, "A geographically weighted regression approach to investigating local built-environment effects on home prices in the housing downturn, recovery, and subsequent increases," Journal of Housing and the Built Environment, vol. 35, no. 1, pp. 1283-1302, 2020.

[31] X. Ma, J. Zhang, C. Ding, and Y. Wang, "A geographically and temporally weighted regression model to explore the spatiotemporal influence of built environment on transit ridership," Computers, Environment and Urban Systems, vol. 70, pp. 113-124, 2018.

[32] D. Salon, "Heterogeneity in the relationship between the built environment and driving: focus on neighborhood type and travel purpose," Research in Transportation Economics, vol. 52, no. 10, pp. 34-45, 2015.

[33] T. Y. Shen, H. C. Yu, L. Zhou, H. Gu, and H. He, "On hedonic price of second-hand houses in Beijing based on multi-scale geographically weighted regression: scale law of spatial heterogeneity," Economic Geography, vol. 40, no. 3, pp. 75-83, 2020.

[34] L. Anselin, "Local indicators of spatial association-LISA," Geographical Analysis, vol. 27, no. 2, pp. 93-115, 1995.

[35] T. M. Oshan, Z. Li, W. Kang, L. J. Wolf, and A. Fotheringham, "MGWR: a Python implementation of multiscale geographically weighted regression for investigating process spatial heterogeneity and scale," International Journal of Geo-Information, vol. 8, no. 6, p. 31, 2019.

[36] W. Tu, R. Cao, Y. Yue, B. Zhou, Q. Li, and Q. Li, "Spatial variations in urban public ridership derived from GPS trajectories and smart card data," Journal of Transport Geography, vol. 69, pp. 45-57, 2018.

[37] Y. Zhuo, M. L. Franz, S. J. Zhu, J. Mahmoudi, A. Nasri, and L. Zhang, "Analysis of Washington, DC taxi demand using GPS and land-use data," Journal of Transport Geography, vol. 66, pp. 35-44, 2018.

[38] T. Munshi, "Built environment and mode choice relationship for commute travel in the city of Rajkot, India," Transportation Research Part D: Transport and Environment, vol. 44, no. 5, pp. 239-253, 2016.

[39] K. Maria Kockelman, "Travel behavior as function of accessibility, land use mixing, and land use balance: evidence from San Francisco bay area," Transportation Research Record: Journal of the Transportation Research Board, vol. 1607, no. 1, pp. 116-125, 1997.

[40] X. Qian and S. V. Ukkusuri, "Spatial variation of the urban taxi ridership using GPS data," Applied Geography, vol. 59, pp. 31-42, 2015. 